\title{
Modesto efeito hipolipemiante do extrato seco de Berinjela (Solanum melongena L.) em mulheres com dislipidemias, sob controle nutricional
}

\author{
Maria da Conceição R. Gonçalves*, Margareth F.F. Melo Diniz, Alexandre Henriques G. \\ Dantas, José Damião C. Borba
}

Laboratório de Tecnologia Farmacêutica “Delby Fernandes de Medeiros”, Universidade Federal da Paraíba, Caixa Postal 5009, 58051-970, João Pessoa, PB, Brasil

\begin{abstract}
RESUMO: Neste estudo, investigou-se o efeito hipolipemiante do extrato seco do fruto de Solanum melongena L. (Berinjela) em pacientes com dislipidemias, sob controle nutricional. Foi realizado no Hospital Universitário Lauro Wanderley/UFPB/PB, constituído de 28 mulheres voluntárias, dislipidêmicas, alocadas para o tratamento com cápsula de Berinjela $(n=14)$ e placebo $(\mathrm{n}=14)$, após assinarem o Termo de Consentimento Livre e Esclarecido. Cada voluntária recebeu diariamente três cápsulas contendo $360 \mathrm{mg}$ de extrato seco de Berinjela ou $360 \mathrm{mg}$ de placebo, em cada cápsula, oralmente, sendo acompanhada mensalmente. Após 3 meses de tratamento, ao se compararem os valores de colesterol total antes e após 90 dias, verificou-se que houve redução dos mesmos $(\mathrm{p}=0,023)$, fato que não ocorreu no grupo controle $(\mathrm{p}=0,778)$. Havendo diferença significativa também nos valores de colesterol HDL $(\mathrm{p}=0,03)$ entre os grupos na avaliação basal e após 90 dias no grupo controle ( $\mathrm{p}=0,026)$. Nos parâmetros hepáticos e renais não foram verificadas diferenças significativas, fora da faixa de normalidade clínica. Concluindo que o extrato seco de Solanum melongena L. (Berinjela), exerce um modesto efeito sobre o perfil lipídico, de pacientes com dislipidemias, não apresentando efeito hepatotóxico, nem reações adversas para quem dela faz uso, salientando, entretanto, que a resposta clínica encontrada neste estudo não atingiu os valores estabelecidos pelas III Diretrizes Brasileiras sobre Dislipidemias e Aterosclerose, necessitando de ensaios clínicos mais aprofundados.
\end{abstract}

Unitermos: Solanum melongena, berinjela, dislipidemia, ensaio clínico.

\begin{abstract}
Modest hypolipidemic effect of Eggplant (Solanum melongena L.) dry extract in women with dislipidemy, under nutritional control". In this research, it has been investigated the hypolipidemic effect of the dry extract of Solanum melongena L. (Eggplant) fruit in patients with dislipidemy, under nutritional control. It was accomplished at the Lauro Wanderley Academical Hospital/UFPB/PB, composed by 28 voluntary women, dislipidemics, selected for the treatment with eggplant's capsule $(n=14)$ and placebo $(n=14)$, after they have signed the free and clear up consentiment term. Each volunteer has received daily three capsules with $360 \mathrm{mg}$ of Eggplant dry extract or $360 \mathrm{mg}$ of placebo, in each capsule, orally and they were monthly accompanied. After three months treatment, when compared the values of full cholesterol before and after 90 days, it was verified that these values were reduced $(\mathrm{p}=0,023)$, fact that did not happened in the control group $(p=0,778)$. There was a significant difference in cholesterol values HDL $(p=0,026)$ among these groups in basal evaluation and after 90 days in the control group $(\mathrm{p}=0,026)$. In hepatic and renal parameters, significant differences were not find out, remaining out of the clinical normality level. Concluding that the dry extract of Solanum melongena L. (Eggplant) has a modest effect over the lipidic profile of patients with dislipidemy, not intervening on other renal parameters, neither presenting toxic hepatic effect nor adverse reactions for those who use it, pointing out, however that the clinical answer found in this study it didn't reach the established values for the Third Brazilian Guidelines on Dislipidemies and Atherosclerosis, needing clinical rehearsals more deepened.
\end{abstract}

Keywords: Solanum melongena, eggplant, dislipidemy, clinical rehearsal.

\section{INTRODUÇÃO}

A doença arterial coronariana (DAC) é a principal causa de morte na maioria dos países desenvolvidos e em muitas nações em desenvolvimento. As complicações clínicas das DACs levam à incapacitação substancial e são a principal fonte da elevação de custos da assistência à saúde. Enquanto a incidência de DAC está diminuindo na Europa Ocidental, EUA e Austrália. Ela está crescendo agudamente na Europa Central e Oriental e, em algum grau, na Ásia e África. Com isto, tornou-se urgente, em todo mundo, a necessidade de estratégias de 
prevenção mais efetivas contra a DAC (Farret, 2005).

Políticas preventivas, tanto em nível populacional quanto individual, vêm sendo desenvolvidas, direcionadas aos fatores de risco clássicos, como idade, sexo, antecedentes pessoais e familiares, não modificáveis e fatores modificáveis de maiores riscos como: hipercolesterolemia, hipertensão e tabagismo.

Os baixos níveis de colesterol HDL, a presença de diabetes mellitus, obesidade e sedentarismo também são vistos como um fator de risco causal para DAC, assim como evidências incluem o aumento dos níveis de triglicerídeos, lipoproteína (a), fribrinogênio, fator de coagulação VIIc (a homocisteína) e o inibidor do ativador do plasminogênio I (plasminogen activator inhibitor I, PAI I) (Graham et al., 1997).

A relação entre a nutrição e a DAC é bem estabelecida e baseia-se em achados epidemiológicos em população e experiências de intervenção nutricional. Hábitos de vida favoráveis à saúde são importantes no controle de fatores de risco e incluem abstenção e/ou suspensão de tabagismo, redução de peso, baixa ingestão de gordura saturada, principalmente trans e aumento de consumo de ácidos graxos poliinsaturados ( $\omega 6$ e $\omega 3)$, além do combate ao sedentarismo.

É importante também a distribuição de gordura corporal, pois o excesso de gordura no tronco e no abdômen é um fator de risco para DAC e têm sua influência adversa sobre os níveis lipídicos, a pressão arterial e a resistência periférica à insulina. Podendo vir a predispor fortemente o desenvolvimento da Síndrome Metabólica, cuja característica principal é obesidade central, hiperinsulinismo e uma ou mais das seguintes anormalidades: dislipidemia, hipertensão arterial sistêmica (HAS), esteatose hepática, gota, hiperuricemia e intolerância à glicose.

A dislipidemia da síndrome metabólica é a "tríade lipídica", consistindo em níveis altos de colesterol LDL e triglicerídeos e níveis baixos de colesterol HDL.

Por que toda dislipidemia deve ser tratada? Porque há uma grande associação da dislipidemia, especialmente a hipercolesterolemia, com o desenvolvimento de aterosclerose, responsável pela maior parte dos casos de infarto do miocárdio e de acidente vascular cerebral, constituindo-se, assim, na principal causa de morte nos Estados Unidos, Europa Ocidental e também no Brasil (Cruz et al., 1998). O tratamento baseia-se primariamente na redução de peso corporal através da dieta e exercícios físicos, isto pode levar a uma acentuada regressão das placas de ateroma e correção de outros desequilíbrios metabólicos, fornecendo indicações claras para o tratamento medicamentoso (Rique et al., 2002).

Muitos tratamentos efetivos com fármacos foram desenvolvidos para combater a hipercolesterolemia. Entretanto, essas drogas hipocolesterolêmicas são de alto custo, com possíveis efeitos colaterais. Tratamentos não farmacológicos, coadjuvantes na prevenção primária como, dieta pobre em gorduras saturadas e colesterol e rica em fibras, exercícios físicos e o não tabagismo têm sido muito utilizado entre a população como um meio de minimizar os efeitos colaterais e os altos custos do tratamento medicamentoso (Gonçalves et al., 2006).

Nesse contexto, destacamos a Solanum melongena L. (Berinjela), planta da família Solanaceae. A berinjela é originária da Índia, sendo seus frutos muito utilizados na alimentação humana. É um vegetal com alto teor de água, baixo de proteínas, é rica em fibras, sais minerais (cálcio, fósforo, potássio e magnésio) e vitaminas (A, $\mathrm{B}_{1}, \mathrm{~B}_{2}$, Niacina e Vitamina C), saponinas, compostos fenólicos, flavonóides e glicoalcalóides (Jeronymo et al., 2000), cuja comercialização na forma de cápsulas contendo extrato seco da planta tem sido utilizado para a redução do colesterol sérico.

Estudos têm sido realizados, principalmente em animais de laboratório (Kritchevsky et al. 1975; Sudheesh et al., 1997; Ribeiro Jorge et al., 1998; Veiga et al., 2000), apontando um papel hipolipemiante para a Solanum melongena $\mathrm{L}$, este aspecto, porém, não é unânime, pois há estudos nos quais não foram observadas melhoras da lipidemia (Silva et al., 1999).

Em humanos, poucos são os trabalhos mostrando este efeito, o primeiro a demonstrá-lo foi Roffo Angel (1943), na Argentina. No Brasil, coube a Cruz et al., 1998, mostrar que esse vegetal tinha o efeito hipolipemiante, usando suco de berinjela com laranja. Discreto efeito hipolipemiante também foi observado pela infusão do pó do fruto de Solanum melongena L. (Berinjela) administrada duas vezes ao dia no estudo de Guimarães et al., 2000, ao contrário dos achados de Kakuta et al., 1997, utilizando suco de berinjela e Silva et al., 2004, utilizando extrato seco, na forma de cápsulas.

Diante destas situações, decidimos empregar um protocolo que simula a utilização comercial do extrato seco, em cápsulas de Solanum melongena L. (Berinjela), em pacientes dislipidêmicos, durante 90 dias, sob controle nutricional.

\section{MATERIAL E MÉTODOS}

\section{Extrato seco de Solanum melongena L (Berinjela) utilizado na preparação das cápsulas}

O extrato seco utilizado nos experimentos foi adquirido da Empresa Ely Martins (lote № 78.251), situada no Município de Ribeirão Preto/São Paulo. Encapsuladas no Laboratório de Tecnologia Farmacêutica da Universidade Federal da Paraíba, em máquina semiautomática e durante o enchimento foram monitorados $\mathrm{o}$ peso e a aparência das cápsulas.

Foram preparadas com o princípio ativo 5400 cápsulas contendo $360 \mathrm{mg}$ de Extrato Seco de Berinjela (Solanum melongena L.) e com o Placebo, também foram preparadas 5400 cápsulas contendo $360 \mathrm{mg}$ de Lactose (excipiente para formas farmacêuticas sólidas), cuja 
Tabela 1. Média e desvio padrão das variáveis relativas ao consumo de alimento segundo o grupo.

\begin{tabular}{llll}
\hline \multirow{2}{*}{ Variável } & \multicolumn{2}{c}{ Grupo } & Valor de $\mathrm{p}$ \\
\cline { 2 - 3 } & \multicolumn{1}{c}{$\begin{array}{c}\text { Experimental } \\
\text { Média } \pm \text { DP }\end{array}$} & $\begin{array}{c}\text { Controle } \\
\text { Média } \pm \mathrm{DP}\end{array}$ & \\
\hline VET consumido (cal) & $1756,54 \pm 549,06$ & $1921,89 \pm 680,88$ & $\mathrm{p}^{(1)}=0,486$ \\
Total de proteínas (g) & $89,93 \pm 32,92$ & $93,17 \pm 41,11$ & $\mathrm{p}^{(1)}=0,820$ \\
Total de calorias de proteínas & $359,73 \pm 131,66$ & $372,67 \pm 164,43$ & $\mathrm{p}^{(1)}=0,820$ \\
\% de proteínas & $20,79 \pm 5,44$ & $19,48 \pm 3,83$ & $\mathrm{p}^{(1)}=0,467$ \\
Total dos carboidratos (g) & $255,38 \pm 102,63$ & $236,81 \pm 77,34$ & $\mathrm{p}^{(1)}=0,593$ \\
Valor de calorias dos HC & $1021,51 \pm 410,53$ & $1664,17 \pm 747,91$ & $\mathrm{p}^{(1)}=0,395$ \\
\% de carboidratos & $57,33 \pm 10,17$ & $50,01 \pm 8,20$ & $\mathrm{p}^{(1)}=0,046^{*}$ \\
Total de lipídeos (g) & $41,70 \pm 15,86$ & $66,89 \pm 33,93$ & $\mathrm{p}^{(2)}=0,018^{*}$ \\
Valor de calorias dos lipídeos & $375,30 \pm 142,78$ & $601,98 \pm 305,37$ & $\mathrm{p}^{(2)}=0,018^{*}$ \\
\% de lipídeos & $21,88 \pm 5,89$ & $30,52 \pm 6,38$ & $\mathrm{p}^{(1)}=0,001^{*}$ \\
\hline
\end{tabular}

(*) - Variância significante a 5,0\%.

(1) - Através do teste t-Student com variâncias iguais.

(2) - Através do teste t-Student com variâncias desiguais.

Tabela 2. Média e desvio padrão das variáveis relativas ao exame físico segundo o grupo.

\begin{tabular}{|c|c|c|c|c|}
\hline \multirow[b]{2}{*}{ Variável } & \multicolumn{3}{|c|}{ Grupo } & \multirow[b]{2}{*}{ Valor de $\mathrm{p}$} \\
\hline & Avaliação & $\begin{array}{l}\text { Experimental } \\
\text { Média } \pm \text { DP }\end{array}$ & $\begin{array}{c}\text { Controle } \\
\text { Média } \pm \mathrm{DP}\end{array}$ & \\
\hline \multirow[t]{5}{*}{ Peso (Kg) } & Basal & $64,53 \pm 6,57$ & $74,66 \pm 7,50$ & $\mathrm{p}^{(1)}=0,001^{*}$ \\
\hline & 30 dias & $64,33 \pm 6,66$ & $73,86 \pm 7,56$ & $\mathrm{p}^{(1)}=0,002^{*}$ \\
\hline & 45 dias & $64,09 \pm 6,64$ & $73,32 \pm 7,43$ & $\mathrm{p}^{(1)}=0,002 *$ \\
\hline & 60 dias & $64,09 \pm 6,50$ & $72,46 \pm 8,08$ & $\mathrm{p}^{(1)}=0,006^{*}$ \\
\hline & 90 dias & $63,76 \pm 6,90$ & $72,12 \pm 8,20$ & $\mathrm{p}^{(1)}=0,007^{*}$ \\
\hline Avaliação dos períodos & & $64,16 \pm 6,63$ & $73.28 \pm 6,68$ & $\mathrm{p}^{(1)}=0,002^{*}$ \\
\hline Altura $(\mathrm{cm})$ & & $1,55 \pm 0,06$ & $1,55 \pm 0,05$ & $\mathrm{p}^{(1)}=1,000$ \\
\hline \multirow[t]{5}{*}{$\operatorname{IMC}\left(\mathrm{Kg} / \mathrm{m}^{2}\right)$} & Basal & $26,95 \pm 3,43$ & $31,01 \pm 3,53$ & $\mathrm{p}^{(1)}=0,005^{*}$ \\
\hline & 30 dias & $26,81 \pm 3,53$ & $30,72 \pm 3,60$ & $\mathrm{p}^{(1)}=0,007^{*}$ \\
\hline & 45 dias & $26,70 \pm 3,64$ & $30,51 \pm 3,66$ & $\mathrm{p}^{(1)}=0,010^{*}$ \\
\hline & 60 dias & $26,73 \pm 3,55$ & $30,13 \pm 3,72$ & $\mathrm{p}^{(1)}=0,020^{*}$ \\
\hline & 90 dias & $26,58 \pm 3,70$ & $30,00 \pm 3,85$ & $\mathrm{p}^{(1)}=0,024^{*}$ \\
\hline Avaliação dos períodos & & $26,75 \pm 3,57$ & $30,48 \pm 3,64$ & $\mathrm{p}^{(1)}=0,001 *$ \\
\hline \multirow[t]{5}{*}{ RCQ (cm) } & Basal & $0,88 \pm 0,06$ & $0,88 \pm 0,04$ & $\mathrm{p}^{(1)}=0,859$ \\
\hline & 30 dias & $0,88 \pm 0,06$ & $0,89 \pm 0,03$ & $\mathrm{p}^{(1)}=0,768$ \\
\hline & 45 dias & $0,88 \pm 0,06$ & $0,88 \pm 0,04$ & $\mathrm{p}^{(1)}=0,889$ \\
\hline & 60 dias & $0,88 \pm 0,06$ & $0,89 \pm 0,03$ & $\mathrm{p}^{(1)}=0,625$ \\
\hline & 90 dias & $0,88 \pm 0,06$ & $0,89 \pm 0,04$ & $\mathrm{p}^{(1)}=0,756$ \\
\hline Avaliação dos períodos & & $0,88 \pm 0,06$ & $0,89 \pm 0,04$ & $\mathrm{p}^{(1)}=0,772$ \\
\hline
\end{tabular}

(*) - Diferença significante a 5,0\%.

(1) - Através do teste t-Student com variâncias iguais.

(2) - Através do teste t-Student com variâncias desiguais.

posologia foi: 3 cápsulas ao dia por 90 dias, ou seja, 270 cápsulas por paciente.

Convém salientar que as cápsulas contendo Solanum melongena L. (Berinjela) ou placebo, bem como os frascos nas quais foram acondicionadas, eram indistinguíveis visualmente.

\section{Estudo Clínico}

16(Supl.):dez. 2006
Foi realizado um estudo clínico de fase II, do tipo randomizado, quantitativo previamente aprovado pelo Comitê de Ética e Pesquisa do Hospital Universitário Lauro Wanderley/HULW (parecer № 326/2004). Com a participação livre e espontânea, de pacientes com dislipidemias após convite realizado pela pesquisadora durante a sua consulta ao Serviço de Nutrição Ambulatorial, e assinatura do termo de consentimento livre e esclarecido, de acordo com as Resoluções № 
Tabela 3. Média e desvio padrão das variáveis relativas ao exame bioquímico (glicemia, TG, CT, HDL, LDL, VLD, VLDL) segundo o grupo, durante as avaliações: basal e 90 dias.

\begin{tabular}{|c|c|c|c|c|}
\hline \multirow[b]{2}{*}{ Variável } & \multicolumn{3}{|c|}{ Grupo } & \multirow[b]{2}{*}{ Valor de $p$} \\
\hline & Avaliação & $\begin{array}{c}\text { Experimental } \\
\text { Média } \pm \text { DP }\end{array}$ & $\begin{array}{c}\text { Controle } \\
\text { Média } \pm \text { DP }\end{array}$ & \\
\hline $\begin{array}{l}\text { Glicemia } \\
(70,0-110,0 \mathrm{mg} / \mathrm{dL})\end{array}$ & $\begin{array}{l}\text { Basal } \\
90 \text { dias } \\
\text { Valor de p }\end{array}$ & $\begin{array}{l}88,21 \pm 12,36 \\
85,86 \pm 14,77 \\
\mathrm{p}^{(3)}=0,438\end{array}$ & $\begin{array}{l}86,29 \pm 13,84 \\
89,21 \pm 8,95 \\
\mathrm{p}^{(3)}=0,490\end{array}$ & $\begin{array}{l}\mathrm{p}^{(1)}=0,700 \\
\mathrm{p}^{(1)}=0,474\end{array}$ \\
\hline $\begin{array}{l}\text { Triglicerídeos } \\
(<200 \mathrm{mg} / \mathrm{dL})\end{array}$ & $\begin{array}{l}\text { Basal } \\
90 \text { dias } \\
\text { Valor de } \mathrm{p}\end{array}$ & $\begin{array}{l}199,00 \pm 96,05 \\
174,36 \pm 75,24 \\
p^{(3)}=0,136\end{array}$ & $\begin{array}{l}162,57 \pm 86,41 \\
158,14 \pm 81,03 \\
p^{(3)}=0,793\end{array}$ & $\begin{array}{l}\mathrm{p}^{(1)}=0,301 \\
\mathrm{p}^{(1)}=0,588\end{array}$ \\
\hline $\begin{array}{l}\text { Colesterol total } \\
(<200 \mathrm{mg} / \mathrm{dL})\end{array}$ & $\begin{array}{l}\text { Basal } \\
90 \text { dias } \\
\text { Valor de } \mathrm{p}\end{array}$ & $\begin{array}{l}230,14 \pm 34,33 \\
213,00 \pm 27,15 \\
\mathrm{p}^{(3)}=0,023^{*}\end{array}$ & $\begin{array}{l}218,36 \pm 21,37 \\
221,14 \pm 25,65 \\
\mathrm{p}^{(3)}=0,778\end{array}$ & $\begin{array}{l}\mathrm{p}^{(1)}=0,285 \\
\mathrm{p}^{(1)}=0,422\end{array}$ \\
\hline $\begin{array}{l}\text { Colesterol HDL } \\
(>35 \mathrm{mg} / \mathrm{dL})\end{array}$ & $\begin{array}{l}\text { Basal } \\
90 \text { dias } \\
\text { Valor de } \mathrm{p}\end{array}$ & $\begin{array}{l}41,64 \pm 11,00 \\
46,43 \pm 11,71 \\
\mathrm{p}^{(3)}=0,235\end{array}$ & $\begin{array}{l}54,50 \pm 9,52^{(\mathrm{A})} \\
46,57 \pm 6,49^{(\mathrm{AB})} \\
\mathrm{p}^{(3)}=0,026^{*}\end{array}$ & $\begin{aligned} \mathrm{p}^{(1)} & =0,003^{*} \\
\mathrm{p}^{(2)} & =0,969\end{aligned}$ \\
\hline $\begin{array}{l}\text { Colesterol LDL } \\
(<130 \mathrm{mg} / \mathrm{dL})\end{array}$ & $\begin{array}{l}\text { Basal } \\
90 \text { dias } \\
\text { Valor de } \mathrm{p}\end{array}$ & $\begin{array}{l}153,33 \pm 43,54 \\
138,30 \pm 47,17 \\
p^{(3)}=0,165\end{array}$ & $\begin{array}{l}131,34 \pm 21,72 \\
143,44 \pm 25,13 \\
p^{(3)}=0,147\end{array}$ & $\begin{array}{l}\mathrm{p}^{(2)}=0,107 \\
\mathrm{p}^{(1)}=0,722\end{array}$ \\
\hline $\begin{array}{l}\text { Colesterol VLDL } \\
(<30 \mathrm{mg} / \mathrm{dL})\end{array}$ & $\begin{array}{l}\text { Basal } \\
90 \text { dias } \\
\text { Valor de } \mathrm{p}\end{array}$ & $\begin{array}{l}39,89 \pm 20,09 \\
34,86 \pm 15,10 \\
\mathrm{p}^{(3)}=0,235\end{array}$ & $\begin{array}{l}32,44 \pm 17,13 \\
31,63 \pm 16,20 \\
\mathrm{p}^{(3)}=0,026^{*}\end{array}$ & $\begin{array}{l}\mathrm{p}^{(1)}=0,309 \\
\mathrm{p}^{(1)}=0,589\end{array}$ \\
\hline
\end{tabular}

(*) - Diferença significante a 5,0\%.

(1) - Através do teste $t$-Student com variâncias iguais.

(2) - Através do teste $t$-Student com variâncias desiguais.

(3) - Através do teste $t$-Student pareado.

196/96 e № 251/97 do Conselho Nacional de Saúde (Brasil, 1996, 1997).

Foram excluídas do estudo as mulheres em uso de medicamento para dislipidemia ou acometidas de Diabetes Mellitus, Síndrome Nefrótica, Insuficiência Renal aguda ou crônica, Hepatopatias, Hipo ou Hipertireoidismo, em reposição hormonal ou outra patologia capaz de alterar o perfil lipídico.

A seleção das pacientes foi baseada nos valores atuais de referência para dislipidemias (hipercolesterolemia e/ou hipertrigliceridemia) de acordo com os dados estabelecidos de referência do colesterol total, as frações colesterol LDL, colesterol HDL e triglicerídeos em adultos, (idade $>18$ anos) (III Diretrizes Brasileiras sobre Dislipidemias e Aterosclerose, 2001).

Foram selecionadas 28 pacientes, das 46 atendidas, com faixa etária entre 18 e 59 anos, distribuídas em dois (02) grupos: Grupo Experimental e Grupo Controle (Placebo). Durante o período do estudo, 90 dias, elas foram acompanhadas clínica e laboratorialmente.

Durante o acompanhamento clínico, realizado a cada 30 dias, as pacientes foram submetidas a uma entrevista para aplicação do Teste de Reação Adversa, aferição de Pressão Arterial, Eletrocardiograma, e medidas de peso e altura, utilizadas para avaliação nutricional baseada no Índice de Massa Corporal (IMC), para avaliar a obesidade (OMS, 1998) e na relação da Cintura-quadril (RCQ), utilizada para predizer o risco cardíaco (Assis, 1997).

A paciente era submetida também a um controle nutricional através da prescrição de uma orientação dietoterápica individual, pobre em gorduras saturadas e colesterol, após a aplicação do Inquérito Recordatório de 24 horas e cálculo do Valor Energético Total (VET), segundo as diretrizes do National Cholesterol Education Program (2001).

O acompanhamento laboratorial foi realizado no Laboratório do Hospital Universitário Lauro Wanderley. A coleta de sangue foi realizada no período da manhã em jejum de 12 horas, em tempo basal $\left(\mathrm{T}_{0}\right), 45$ dias $\left(\mathrm{T}_{45}\right)$ e 90 dias $\left(\mathrm{T}_{90}\right)$, para todas as variáveis, incluindo as de função hepática e renal, para avaliação de efeitos tóxicos.

Fechado o grupo experimental, foi formado o grupo placebo ou controle, no qual elas receberam 
Tabela 4. Média e desvio padrão das variáveis relativas ao exame bioquímico segundo o grupo.

\begin{tabular}{|c|c|c|c|c|}
\hline \multirow[b]{2}{*}{ Variável } & \multicolumn{3}{|c|}{ Grupo } & \multirow[b]{2}{*}{ Valor de $\mathrm{p}$} \\
\hline & Avaliação & $\begin{array}{l}\text { Experimental } \\
\text { Média } \pm \text { DP }\end{array}$ & $\begin{array}{c}\text { Controle } \\
\text { Média } \pm \text { DP }\end{array}$ & \\
\hline - Fosfatase alcalina & $\begin{array}{l}\text { Basal } \\
45 \text { dias }\end{array}$ & $\begin{array}{l}74,50 \pm 27,33 \\
81,43 \pm 35,44\end{array}$ & $\begin{array}{l}68,14 \pm 21,67 \\
66,79 \pm 21,77\end{array}$ & $\begin{array}{l}p^{(1)}=0,501 \\
p^{(1)}=0,199\end{array}$ \\
\hline$(30$ a $100 \mathrm{U} / \mathrm{L})$ & $\begin{array}{l}90 \text { dias } \\
\text { Valor de } \mathrm{p}\end{array}$ & $\begin{array}{c}80,50 \pm 25,19 \\
p^{(1)}=0,310\end{array}$ & $\begin{array}{c}72,86 \pm 27,05 \\
\mathrm{p}^{(1)}=0,551\end{array}$ & $p^{(1)}=0,446$ \\
\hline - Bilirrubina total & $\begin{array}{l}\text { Basal } \\
45 \text { dias }\end{array}$ & $\begin{array}{l}0,67 \pm 0,33 \\
0,60 \pm 0,19\end{array}$ & $\begin{array}{l}0,64 \pm 0,21 \\
0,54 \pm 0,21\end{array}$ & $\begin{array}{l}\mathrm{p}^{(1)}=0,719 \\
\mathrm{p}^{(1)}=0,411\end{array}$ \\
\hline (até $1,2 \mathrm{mg} / \mathrm{dl}$ ) & $\begin{array}{l}90 \text { dias } \\
\text { Valor de p }\end{array}$ & $\begin{array}{l}0,61 \pm 0,38 \\
\mathrm{p}^{(1)}=0,650\end{array}$ & $\begin{array}{l}0,54 \pm 0,29 \\
\mathrm{p}^{(1)}=0,424\end{array}$ & $\mathrm{p}^{(1)}=0,544$ \\
\hline - Bilirrubina direta & $\begin{array}{l}\text { Basal } \\
45 \text { dias }\end{array}$ & $\begin{array}{l}0,18 \pm 0,10 \\
0,16 \pm 0,08\end{array}$ & $\begin{array}{l}0,25 \pm 0,15 \\
0,16 \pm 0,09\end{array}$ & $\begin{array}{l}p^{(1)}=0,151 \\
p^{(1)}=0,826\end{array}$ \\
\hline (até 0,4 mg/dl) & $\begin{array}{l}90 \text { dias } \\
\text { Valor de } \mathrm{p}\end{array}$ & $\begin{array}{l}0,17 \pm 0,10 \\
\mathrm{p}^{(1)}=0,839\end{array}$ & $\begin{array}{l}0,18 \pm 0,16 \\
\mathrm{p}^{(1)}=0,232\end{array}$ & $\mathrm{p}^{(1)}=0,856$ \\
\hline $\begin{array}{l}\text { - Bilirrubina indireta } \\
\text { (até } 0,8 \mathrm{mg} / \mathrm{dl} \text { ) }\end{array}$ & $\begin{array}{l}\text { Basal } \\
45 \text { dias } \\
90 \text { dias } \\
\text { Valor de p }\end{array}$ & $\begin{array}{l}0,48 \pm 0,32 \\
0,43 \pm 0,19 \\
0,45 \pm 0,40 \\
p^{(1)}=0,811\end{array}$ & $\begin{array}{l}0,38 \pm 0,16 \\
0,37 \pm 0,16 \\
0,36 \pm 0,19 \\
p^{(1)}=0,914\end{array}$ & $\begin{array}{l}p^{(1)}=0,318 \\
p^{(1)}=0,378 \\
p^{(1)}=0,468\end{array}$ \\
\hline $\begin{array}{l}\text { - Gama GT } \\
\text { (7 a } 32 \mathrm{U} / \mathrm{L})\end{array}$ & $\begin{array}{l}\text { Basal } \\
45 \text { dias } \\
90 \text { dias } \\
\text { Valor de p }\end{array}$ & $\begin{array}{c}23,36 \pm 10,94^{(\mathrm{AB})} \\
21,79 \pm 9,68^{(\mathrm{A})} \\
26,29 \pm 14,29^{(\mathrm{B})} \\
\mathrm{p}^{(1)}=0,025^{*}\end{array}$ & $\begin{array}{c}28,86 \pm 10,33 \\
25,21 \pm 8,28 \\
26,71 \pm 10,83 \\
p^{(1)}=0,061\end{array}$ & $\begin{array}{l}\mathrm{p}^{(1)}=0,183 \\
\mathrm{p}^{(1)}=0,323 \\
\mathrm{p}^{(1)}=0,929\end{array}$ \\
\hline $\begin{array}{l}\text { - Creatinina } \\
(0,4 \text { até } 1,2 \mathrm{mg} / \mathrm{dl})\end{array}$ & $\begin{array}{l}\text { Basal } \\
45 \text { dias } \\
90 \text { dias } \\
\text { Valor de p }\end{array}$ & $\begin{array}{l}0,79 \pm 0,19 \\
0,67 \pm 0,13 \\
0,66 \pm 0,13 \\
\mathrm{p}^{(1)}=0,059\end{array}$ & $\begin{array}{l}0,76 \pm 0,12 \\
0,72 \pm 0,15 \\
0,68 \pm 0,18 \\
\mathrm{p}^{(1)}=0,229\end{array}$ & $\begin{array}{l}p^{(1)}=0,723 \\
p^{(1)}=0,345 \\
p^{(1)}=0,724\end{array}$ \\
\hline - Ácido úrico & $\begin{array}{l}\text { Basal } \\
45 \text { dias }\end{array}$ & $\begin{array}{l}4,54 \pm 1,09^{(\mathrm{A})} \\
5,65 \pm 1,51^{(\mathrm{B})}\end{array}$ & $\begin{array}{l}4,82 \pm 0,99^{(\mathrm{A})} \\
3,72 \pm 0,97^{(\mathrm{B})}\end{array}$ & $\begin{array}{c}\mathrm{p}^{(1)}=0,473 \\
\mathrm{p}^{(1)}=0,001^{*}\end{array}$ \\
\hline$(2,5$ a $6,2 \mathrm{mg} / \mathrm{dl})$ & $\begin{array}{l}90 \text { dias } \\
\text { Valor de } \mathrm{p}\end{array}$ & $\begin{aligned} 4,70 & \pm 1,49^{(\mathrm{AB})} \\
\mathrm{p}^{(1)} & =0,014^{*}\end{aligned}$ & $\begin{array}{c}3,51 \pm 1,05^{(\mathrm{B})} \\
\mathrm{p}^{(1)}=0,001^{*}\end{array}$ & $\mathrm{p}^{(1)}=0,021^{*}$ \\
\hline $\begin{array}{l}\text { - Uréia } \\
\text { (10 a } 50 \mathrm{mg} / \mathrm{dl})\end{array}$ & $\begin{array}{l}\text { Basal } \\
45 \text { dias } \\
90 \text { dias }\end{array}$ & $\begin{array}{l}26,36 \pm 7,47 \\
26,79 \pm 5,55 \\
28,00 \pm 7,53\end{array}$ & $\begin{array}{l}28,21 \pm 8,06 \\
29,29 \pm 7,05 \\
29,86 \pm 6,13\end{array}$ & $\begin{array}{l}p^{(1)}=0,533 \\
p^{(1)}=0,307 \\
p^{(1)}=0,481\end{array}$ \\
\hline - $\mathrm{AST}$ & $\begin{array}{l}\text { Basal } \\
45 \text { dias }\end{array}$ & $\begin{array}{c}29,64 \pm 9,52 \\
33,00 \pm 12,73\end{array}$ & $\begin{array}{l}29,71 \pm 7,87 \\
28,79+5,99\end{array}$ & $\begin{array}{l}\mathrm{p}^{(1)}=0,983 \\
\mathrm{p}^{(1)}=0,273\end{array}$ \\
\hline$(7$ a $45 \mathrm{U} / \mathrm{L})$ & $\begin{array}{l}90 \text { dias } \\
\text { Valor de } \mathrm{p}\end{array}$ & $\begin{array}{c}30,29 \pm 8,33 \\
\mathrm{p}^{(1)}=0,594\end{array}$ & $\begin{array}{c}25,64 \pm 4,92 \\
\mathrm{p}^{(1)}=0,164\end{array}$ & $\mathrm{p}^{(1)}=0,084$ \\
\hline - ALT & $\begin{array}{l}\text { Basal } \\
45 \text { dias }\end{array}$ & $\begin{array}{c}26,14 \pm 11,39 \\
26,71 \pm 9,25\end{array}$ & $\begin{array}{l}28,85 \pm 11,50^{(\mathrm{A})} \\
24,38 \pm 7,54^{(\mathrm{AB})}\end{array}$ & $\begin{array}{l}p^{(1)}=0,545 \\
p^{(1)}=0,482\end{array}$ \\
\hline (até $49 \mathrm{U} / \mathrm{L}$ ) & $\begin{array}{l}90 \text { dias } \\
\text { Valor de } \mathrm{p}\end{array}$ & $\begin{array}{c}27,86 \pm 12,14 \\
p^{(1)}=0,872\end{array}$ & $\begin{array}{c}22,31 \pm 6,61 \\
\mathrm{p}^{(1)}=0,035^{*}\end{array}$ & $\mathrm{p}^{(1)}=0,157$ \\
\hline
\end{tabular}

$(*)$ - Diferença significante a 5,0\%.

(1) - Através do teste t-Student com variâncias iguais. 
cápsulas de aspecto idêntico mantendo o mesmo protocolo.

O tratamento foi realizado através da ingestão de cápsulas contendo $360 \mathrm{mg}$ de extrato seco do fruto de Berinjela roxa (Solanum melongena L.) durante 13 semanas (90 dias), para ser ingerido três (03) vezes ao dia antes das refeições, com a primeira dose em jejum.

\section{Análise Estatística}

Para análise dos dados de distribuições absolutas, variáveis nominais e medidas estatísticas foram utilizadas as técnicas de Estatística Descritiva e utilizados os testes: t-Student pareado com variâncias iguais ou desiguais e o teste F (ANOVA) com medidas repetidas. No caso de diferença significante entre as médias das avaliações foram utilizados os testes de comparações pareadas de Bonferroni. O nível de significância utilizado na decisão dos testes estatísticos foi de 5\% $(0,05)$. O software utilizado para a obtenção dos cálculos estatísticos foi o SPSS (Statistical Package for the Social Sciences) na versão 11 (Jerrold, 1999).

\section{RESULTADOS}

Dentre as 28 pacientes voluntárias com idade de 23 a 56 anos, que fizeram parte do estudo, todas cumpriram integralmente os 90 dias, apresentando um perfil homogêneo para os parâmetros analisados, entre eles, hipercolesterolemia e/ou hipertrigliceridemia.

Com relação ao controle nutricional, na análise do padrão alimentar, pode-se verificar que houve diferença significante entre os dois grupos quanto ao percentual de carboidratos $(\mathrm{p}=0,046)$, total de lipídeos $(\mathrm{g})$ $(\mathrm{p}=0,018)$, percentual de lipídeos $(\mathrm{p}=0,001)$ e seu valor calórico correspondente $(\mathrm{p}=0,018)$ (Tabela 1$)$, havendo maior presença de obesidade e sobrepeso, neste grupo, com a média do IMC de $30,48 \mathrm{~kg} / \mathrm{m}^{2}$ (Tabela 2).

Ao compara-se os valores médios do colesterol e suas frações (HDL, LDL, VLDL e TG), após 90 dias, no grupo experimental, observou-se que houve redução em todos os parâmetros analisados, significativo para o colesterol total $(p=0,023)$, com exceção da fração HDL que mostrou um aumento não significativo $(\mathrm{p}=0,235)$. No grupo controle, pode-se observar que houve uma diminuição dos valores médios não significativos para os triglicerídeos e significativo $(\mathrm{p}=0,026)$ para as frações HDL e VLDL, com aumento nos valores médios do colesterol total e fração LDL não significativo (Tabela $3)$.

No que concerne aos exames bioquímicos, para a avaliação da função hepática e renal, não se comprova diferença significativa entre os grupos, para as variáveis: AST, ALT, fosfatase alcalina, $\gamma \mathrm{GT}$ e bilirrubinas. Nem para creatinina e uréia, havendo um aumento significativo entre os grupos para o ácido úrico, na avaliação de 45 dias $(\mathrm{p}=0,001)$ e de 90 dias $(\mathrm{p}=0,021)$, porém, sem assumir relevância clínica, uma vez que estes valores se encontram dentro da faixa de normalidade bioquímica $(2,5$ a $6,2 \mathrm{mg}$ / dl) (Tabela 4).

Ao aplicar-se o questionário das reações adversas, observou-se que não houve efeito adverso significativo após uso das cápsulas.

\section{DISCUSSÃO}

De acordo com as últimas Diretrizes Brasileiras sobre Dislipidemias e Aterosclerose (2001), os fatores de risco mais evidentes no panorama da saúde cardiovascular no Brasil são: tabagismo, hipertensão arterial sistêmica (HAS), diabetes mellitus, obesidade mórbida e dislipidemias, esses fatores ficaram bem evidenciados no presente estudo, pois, a maioria das pacientes tinha hipertensão arterial sistêmica (HAS), algumas apresentavam obesidade e todas tinham dislipidemias, com antecedentes familiares também na sua maioria, cardíacos, hipertensos e diabéticos, corroborando com os estudos de Marcopito et al. (2005), Viebig et al. (2006) e Neumann et al. (2006).

A nutrição adequada pode alterar a incidência e a gravidade das doenças coronarianas, inclusive a aterosclerose, que tem como fator de risco o consumo de gorduras saturadas e colesterol. Embora ainda existam controvérsias, a redução de lipídeos para o máximo de $35 \%$ do valor energético total (VET) já resulta em benefícios no controle de risco das DVC. Os lipídeos que mais contribuem para o aumento da LDC-C são os ácidos graxos saturados, os ácidos graxos transisômeros, e, em menor extensão, o colesterol dietético (Otten et al., 2006).

No estudo, o grupo experimental tinha o hábito de consumir menos lipídeos na sua alimentação do que no grupo controle, apresentando um valor calórico maior oriundo dos lipídeos. Isto também refletiu na média do peso dessas pacientes ser mais alto no grupo controle do que no experimental, em que a avaliação nutricional mostrou sobrepeso com maior destaque, para este grupo.

A primeira demonstração conhecida de que a berinjela (Solanum melongena L.) seria capaz de baixar o colesterol sangüíneo e reduzir a ação das gorduras sobre o fígado aconteceu em 1943, pelo professor Angel H. Roffo, na Argentina, usando-a in natura na forma de suco. A sua ação acontece apenas sobre a fração LDL, não agindo nos triglicerídeos, nem na fração HDL.

No Brasil, em 1998, Ribeiro Jorge et al. estudaram o efeito do suco da berinjela sobre os lipídeos plasmáticos, o colesterol tecidual, a peroxidação lipídica das LDL nativas, oxidadas e da parede arterial e o relaxamento dependente do endotélio, em coelhos hipercolesterolêmicos. Concluindo que o suco da berinjela administrado nos animais reduziu significativamente o peso corpóreo, o colesterol total (19\%), as LDL colesterol plasmáticas (29\%) e os triglicerídeos em 38\%. Reduziu também o colesterol 
tecidual, a peroxidação lipídica das LDL nativas, oxidadas e da parede arterial, assim como aumentou o relaxamento dependente do endotélio.

A partir de então, estudos pré-clínicos (Mitscheck, 1975, Silva et al., 1999; Veiga et al., 2000), clínicos (Cruz et al., 1998), de toxicidade pré-clinica (Citra et al., 2000) e clínica (Guimarães et al. 2000) têm sido investigados, em busca de resposta para sua viabilidade terapêutica, cientificamente comprovada.

No presente estudo ao compara-se os valores de colesterol total e suas frações antes e após 90 dias de tratamento com as cápsulas de Berinjela, tempo estabelecido suficiente para confirmar o efeito hipolipemiante (Silva et al., 2004); verificou-se que no grupo experimental todos os valores médios estavam menores do que na avaliação basal, com destaque para o colesterol total $(\mathrm{p}=0,023)$, e aumento não significativo $(\mathrm{p}=0,235)$ para fração HDL. Ao contrário no grupo controle os valores médios aos 90 dias eram mais altos para o colesterol total e LDL, com redução significativa $(=0,026)$ para as frações HDL e VLDL e não significativo para os triglicerídeos.

Isto nos leva a concluir que o extrato seco de Solanum melongena L. (Berinjela), na forma de cápsula, exerce um modesto efeito hipolipemiante, sob controle nutricional, na amostra estudada, não apresentando efeito hepatótoxico, corroborando com o estudo de Guimarães et al. (2000), que observa este mesmo efeito ao testar o extrato seco em forma de infusão.

Outros estudos clínicos também realizados, no entanto, não estão de acordo com os resultados encontrados, como o de Kakuda et al., 1997, que não encontraram efeito hipolipemiante do suco da berinjela liquidificada com laranja, com o estudo realizado por Silva et al. 2004, ao investigar o potencial hipolipemiante do extrato seco do fruto de berinjela e o de Praça et al., 2004, ao avaliarem o efeito do suco de berinjela sobre os lípides plasmáticos em comparação o uso da lovastatina.

Logo, se faz pertinente avaliar o diferencial metodológico entre os estudos, principalmente no que se refere ao tamanho da amostra, tempo e dosagem utilizada, pois, existem informações indicando que preparações de berinjela (Solanum melongena L), na sua forma in natura ou na sua forma seca, reduzem o colesterol sangüíneo em animais, principalmente coelhos. $\mathrm{O}$ que poderia ser provavelmente justificado, pelo fato de que estas investigações envolvem modelos experimentais animais ou empregam frações ou substâncias isoladas, condições nas quais o princípio ativo está mais concentrado em relação ao extrato seco.

\section{CONCLUSÃO}

Portanto, o presente estudo sugere que os benefícios do extrato seco de Solanum melongena L. (Berinjela) sob o controle da dieta com baixo teor de gorduras saturadas e colesterol sobre o perfil lipídico, é uma opção terapêutica viável para a prevenção primária de portadores de Doenças Cardiovasculares, principalmente as Dislipidemias. Salientando, entretanto que, a resposta clínica encontrada neste estudo, não atingiu os valores de normalidade estabelecidos pelas III Diretrizes Brasileiras sobre Dislipidemias e Aterosclerose, necessitando de ensaios clínicos mais aprofundados.

\section{REFERÊNCIAS}

Assis MAA 1997. Consulta de Nutrição: controle e prevenção do colesterol elevado. Ed. Insular, Florianópolis, $168 \mathrm{p}$.

Brasil 1996. Resolução 196/96 do Conselho Nacional de Saúde. Dispõe sobre pesquisa envolvendo seres humanos. Brasília, DF: Comissão Nacional de Ética em Pesquisa (CONEP).

Brasil 1997. Resolução No 251/97 do Conselho Nacional de Saúde. Dispõe sobre as normas de pesquisa envolvendo seres humanos para a área temática de pesquisa com novos fármacos, medicamentos, vacinas e testes diagnósticos: Brasília, DF: Ministerio da Saúde.

Citra DEC, Carvalho JCT, Caputo LRG, Vicita WA 2000. Atividade hipocolesterolêmica e toxicidade em fase de tratamento sub-crônico do extrato hidroalcólico de Solanum melongena (Berinjela). Farmacotécnica e controle de qualidade. Anais do XV Reunião Atualidade de Sociedade de Biologia Experimental.

Cruz J, Cruz HMM, Barbosa-Filho JM 1998. Tratamento das Hiperlipidemias. In: Jenner Cruz, Rui Toledo Barros, Helga Maria Mazzarolo Cruz (Org.). Atualidades em Nefrologia 5. Ed. Sarvier. São Paulo v.5, p. 460-467.

III Diretrizes Brasileiras sobre Dislipidemias e Aterosclerose 2001. Publicação do Departamento de Aterosclerose da Sociedade Brasileira de Cardiologia. Data final de elaboração da versão: 23 de agosto de 2001.

Farret JF 2005. Nutrição e Doenças Cardiovasculares. Prevenção Primária e Secundária. Ed. Atheneu. São Paulo, 245p.

Gonçalves MCR, Diniz MFFM, Borba JDC, Nunes XP, Barbosa-Filho JM 2006. Berinjela (Solanum melongena L.) - mito ou realidade no combate as dislipidemias? Rev Bras Farmacogn 16: 252-257.

Graham IM, Daly LE, Refsum HM, Robinson K, Brattström LE, Ueland PM, Palma-Reis RJ, Boers GH, Sheahan $\mathrm{RG}$, Israelsson $\mathrm{B}$, Uiterwaal $\mathrm{CS}$, Meleady R, McMaster D, Verhoef P, Witteman J, Rubba P, Bellet H, Wautrecht JC, de Valk HW, Sales-Lúis AC, ParrotRouland FM, Tan KS, Higgins I, Garcon D, Andria $\mathrm{G}$ 1997. Plasma homocysteine as a risk factor for vascular disease - The European Concerted Action Project. JAMA 277: 1775-1781.

Guimarães PR, Galvão AMP, Batista CM, Azevedo GS, Oliveira RD, Lamounier RP, Freire N, Barros AMD, Sakurai E, Oliveira JP, Vieira EC, Alvarez-Leite JI 2000. Eggplant (Solanum melongena) infusion has a modest and transitory effect on hypercholesterolemic subjects. Bras J Med Biol Res 33: 1027-1036.

Jeronymo K, Klein AM, Brandão MGL 2000. Preparação e avaliação das fórmulas caseiras e oficinais à base de Berinjela (Solanum melongena L.) usadas no tratamento de hipercolesterolemias. Farmacotécnica e 
controle de qualidade. Anais do XV Reunião Atualidade de Sociedade de Biologia Experimental.

Jerrold HZ 1999. Biostatistical Analysis. Four Edition. Prentice Hall, New Jersey,USA, 929 p.

Kakuda CM, Aoki L, Ferrari MA, Lotierzo PH, Caramelli B 1997. Influence of a eggplant and orange juice on lipids and fibrinogen. Atherosclerosis 134: 325.

Kritchevsky D, Tepper AS, Story JA 1975. Influence of an eggplant (Solanum melongena) preparation on cholesterol metabolism in rats. Exp Pathol Jena 10: 180-183.

Marcopito LF, Rodrigues SSF, Pacheco MA, Shirassu MM, Goldfeder AJ, Moraes MA 2005. Prevalência de alguns fatores de risco para doenças crônicas na cidade de São Paulo. Rev Saúde Pública 39: 738-745.

Mitschek GH 1975. Effect of Solanum melongena on experimental atheromatosis. IV. Histological studies on cholesterol induced atheromatosis in rabbits in mean and long-term test. Exp Pathol Jena 10: 156166.

National Cholesterol Education Program 2001. Executive summary of the third report of the NCEP expert panel on detection, evaluation and treatment of high blood cholesterol in adults (adult treatment panel III). JAMA 285: 2486-2496.

Neumann AICP, Shirassu MM, Fisberg RM 2006. Consumo de alimentos de risco e proteção para doenças cardiovasculares entre funcionários públicos. Rev Nutr 19: 19-28.

Organización Mundial de la Salud 1998. Necesidades de Energia y de Proteinas. Ginebra, 225p. (Informe de una Reunion consultiva conjunta FAO/OMS/ONU de expertos: Série de Informes Técnicos n. 724).

Otten JI, Hellwing JP, Meyers LD 2006. Dietary Reference Intakes: the essential guide to nutrient requeriments. Institute of Medicene of the National Academic, Washington, D.C., 529p.

Praça JM, Thomaz A, Caramelli B 2004. Eggplant (Solanum melongena) extract does not alter serum lipid levels. Arq Bras Cardiol 82: 269-276.

Ribeiro Jorge PAR, Neyra LC, Osaki RM, Almeida E, Bragagnolo N 1998. Efeito da berinjela sobre os lípides plasmáticos, a peroxidação lipídica e a reversão da disfunção endotelial na hipercolesterolemia experimental. Arq Bras Cardiol 70: 87-91.

Rique ABR, Soares EA, Meirelles CM 2002. Nutrição e exercício na prevenção e controle das doenças cardiovasculares. Rev Bras Med Esporte 8: 243-253.

Roffo HA 1943. La berenjena como descolesterimizante (Solanum melongena). Bol Ins Med Exp Estud Trat Câcer 20: 515-578.

Silva M, Santos RC, O'leary MC, Santos RS 1999. Effect of aubergiene (Solanum melongena) on serum and hepatic cholesterol and triglycerides in rats. Braz Arch Biol Technol 42: 339-342.

Silva GEC, Takahashi MH, Eik-Filho W, Albino CC, Tasim GE, Serri LAF, Assef AH, Cortez DAG, Bazotte RB 2004. Ausência de efeito hipolipemiante da Solanum melongena L. (Berinjela) em pacientes hiperlipidêmicos. Arq Bras Endocrinol Metabol 48: 368-373.

Sudheesh S, Presannakumar G, Vijayakumar S, Vijayalakshmi NR 1997. Hypolipidemic effect of flavonoids from
Solanum melongena. Plant Foods Human Nutr 51: 321-330.

Veiga MAC, Utida L, Crespigio J, Nascimento M, Nisioka R, Rodrigues T, Yamada M, Aoki S, Nishibe S, Chiarelli L, Santos F, Siqueira L, Teixaira O, Morozin Zaia, DAM, Zaia CTBV 2000. Extrato bruto aquoso de Solanum melongena (Berinjela) diminui o colesterol plasmático de ratos. Farmacotécnica e controle de qualidade. Anais do XV Reunião Atualidade de Sociedade de Biologia Experimental.

Viebig RF, Valero MP, Araújo F, Yamada AT, Mansur AJ 2006. Perfil de saúde cardiovascular de uma população adulta da região metropolitana de São Paulo. Arq Bras Cardiol 86: 353-360. 